\title{
M2 macrophages reduce the radiosensitivity of head and neck cancer by releasing HB-EGF
}

\author{
ENHUI FU ${ }^{1}$, TIANYANG LIU ${ }^{1}$, SIYANG YU ${ }^{1}$, XIAOHANG CHEN ${ }^{2}$, \\ LIANHAO SONG ${ }^{1}$, HUIHUANG LOU ${ }^{3}$, FEN MA $^{1}$, SIWEI ZHANG ${ }^{1}$, SAJJAD HUSSAIN ${ }^{1}$, \\ JUNNAN GUO ${ }^{4}$, JI SUN ${ }^{4}$, PINGYANG YU ${ }^{4}$, XIONGHUI MAO ${ }^{4}$ and LANLAN WEI ${ }^{1}$ \\ ${ }^{1}$ Department of Microbiology, Harbin Medical University, Wu Lien-Teh Institute, \\ The Heilongjiang Key Laboratory of Immunity and Infection, Harbin, Heilongjiang 150081; \\ ${ }^{2}$ The Genetics Laboratory, Longgang District Maternity and Child Healthcare Hospital, Shenzhen, Guangdong 518100; \\ ${ }^{3}$ The Second Department of Microbiological Examination, Center for Disease Control and Prevention in Wenzhou, \\ Wenzhou Zhejiang 325000; ${ }^{4}$ Department of Head and Neck Surgery, The Tumor Hospital of HMU, \\ Harbin, Heilongjiang 150080, P.R. China
}

Received October 12, 2019; Accepted April 24, 2020

DOI: $10.3892 /$ or.2020.7628

\begin{abstract}
The aim of the present study was to examine the potential role of human heparin-binding epidermal growth factor (HB-EGF) secreted by M2 macrophages in the development of radioresistance in head and neck squamous cell carcinoma (HNSCC). Immunohistochemistry was used to detect radiosensitivity in human papilloma virus (HPV)-positive and HPV-negative HNSCC tissues and immunohistochemical staining with specific antibodies for macrophage surface markers was used to assess the infiltration of M1 and M2 macrophages in HPV-positive and -negative HNSCC tissues. The expression of HB-EGF in HPV-positive and -negative HNSCC tissues was determined by multi-cytokine detection in order to determine the relationship between HB-EGF and radiosensitivity. M1 and M2 macrophages were co-cultured with the HNSCC cell line CAL27 and treated with HB-EGF and its neutralizing antibodies to assess radiation sensitivity. Finally, the major DNA double-strand break repair pathways required for the activation of HB-EGF and promotion of epidermal growth factor receptor (EGFR) were
\end{abstract}

Correspondence to: Professor Lanlan Wei, Department of Microbiology, Harbin Medical University, Wu Lien-Teh Institute, The Heilongjiang Key Laboratory of Immunity and Infection, 157 Baojian Road, Harbin, Heilongjiang 150081, P.R. China E-mail: 3105272165@qq.com

Dr Xionghui Mao, Department of Head and Neck Surgery, The Tumor Hospital of HMU, 150 Haping Road, Harbin, Heilongjiang 150080, P.R. China

E-mail: 15714642@qq.com

Key words: head and neck squamous cell carcinoma, human papillomavirus, radiosensitivity, M2 macrophages, heparin-binding epidermal growth factor identified. The results revealed that radiosensitivity was higher in HPV-positive HNSCC compared with HPV-negative. There was a higher infiltration of M2 macrophages in HPV-negative HNSCC, which were revealed as the main source of HB-EGF secretion. Furthermore, it was determined that overexpression of HB-EGF induced radioresistance in HPV-negative HNSCC. HB-EGF promoted the activation of the non-homologous end-joining pathway by activating EGFR. To the best of our knowledge, this is the first study to demonstrate the association between HB-EGF and radiosensitivity in HNSCC. These results indicated that the secretion of HB-EGF by M2 macrophages could induce radioresistance of HPV-negative HNSCC.

\section{Introduction}

Head and neck squamous cell carcinoma (HNSCC) is the seventh most common cancer in the world, with $\sim 600,000$ cases each year. The 5-year survival rate is generally $<50 \%(1,2)$. HNSCC is divided into two types, human papilloma virus (HPV)-positive and HPV-negative, which is dependent on whether HNSCC is caused by HPV (3). Due to the special anatomical location of HNSCC, radiotherapy is one of the most important treatment for patients with advanced $\operatorname{HNSCC}(4,5)$. Unfortunately, $~ 50 \%$ of patients with HNSCC are insensitive to radiation therapy and therefore do not survive, suggesting that HNSCC is resistant to radiation. HPV-negative HNSCC is more radioresistant than HPV-positive $(6,7)$. However, the cause of radioresistance in HPV-negative HNSCC remains unclear. Therefore, it is critical to investigate the mechanisms of radioresistance in HPV-negative HNSCC in order to improve the survival rates of patients.

The present findings indicated that a potential cause of radioresistance in patients with HPV-negative HNSCC is M2 macrophages. With the renaissance of tumor immunotherapy in recent years, the immune system is considered to be a major regulator of the tumor microenvironment (TME) in HNSCC (8) Numerous researchers have proposed the important role of 
TME macrophages in radiotherapy $(9,10)$. Tumor-associated macrophages (TAMs) are a key component of TME and play an important role in accelerating cancer progression (11). TAMs are divided into M1 type with antitumor activity and M2 type with tumor invasion and metastasis activity (12). Moreover, a number of studies have suggested that the high density of M2 macrophages in tumors is significantly associated with poor prognosis in patients with HNSCC (13-16). It was reported that the low ratio of M1 to M2 macrophages was associated with radiosensitivity of HPV-positive HNSCC (17); however, its mechanism of action remains unclear. Some researchers have demonstrated that macrophages can enhance the DNA damage response of nearby cells by releasing human heparin-binding epidermal growth factor (HB-EGF) (18). However, it is unclear which macrophages are the main source of HB-EGF. In the present study, potential targets related to radioresistance were screened in HNSCC clinical samples from The Cancer Genome Atlas (TCGA) database and experimental procedures. It was hypothesized that M2 macrophages can enhance DNA damage repair via the HB-EGF-mediated epidermal growth factor receptor (EGFR) pathway. These findings may reveal a novel immune cell-mediated system that could contribute to increase the resistance of HNSCC cells to DNA damage at the tissue level.

\section{Materials and methods}

Cell culture and co-culture. An HPV-positive cell line (SCC090) was purchased from The Cell Bank of Type Culture Collection of the Chinese Academy of Sciences. An HPV-negative cell line (CAL27) was provided by Professor $\mathrm{Fu}$ (Harbin Medical University). The human monocytic cell line (THP1) was obtained from the authors' stock at Harbin Medical University. SCC090 cells were maintained in $89 \%$ McCoy's 5A (Gibco; Thermo Fisher Scientific, Inc.) and THP1 cells were maintained in 89\% RPMI-1640 (Gibco; Thermo Fisher Scientific, Inc.), which was supplemented with $9 \%$ fetal bovine serum (CellMax) and $1 \%$ penicillin in a $37^{\circ} \mathrm{C}$ atmosphere containing $5 \% \mathrm{CO}_{2}$.

THP1 cells were cultured in $0.4-\mu \mathrm{m}$-pore-size Transwells (Corning Inc.), in which $1.5 \times 10^{5}$ cells were seeded and cultured in each chamber containing $100 \mathrm{ng} / \mathrm{ml}$ PMA (cat. no. 16561-29-8; Sigma-Aldrich; Merck KGaA) for $24 \mathrm{~h}$ at $37^{\circ} \mathrm{C}$. Then, $100 \mathrm{ng} / \mathrm{ml}$ lipopolysaccharides (cat. no. L8880; Solarbio Life Sciences) and $20 \mathrm{ng} / \mathrm{ml}$ interferon- $\gamma$ (cat. no. 300-02-20UG; PeproTech, Inc.) or $20 \mathrm{ng} / \mathrm{ml}$ interleukin (IL)-4 (cat. no. 96-200-04-5; PeproTech, Inc.) and $20 \mathrm{ng} / \mathrm{ml}$ IL-13 (cat. no. 96-200-13-2; PeproTech, Inc.) were added to induce the polarization of THP1 cells to M1 or M2, respectively. After a $48 \mathrm{~h}$-polarizing process of THP1, 2x10 5 CAL27 cells were seeded and co-cultured in each basal chamber for $24 \mathrm{~h}$, followed by $20 \mathrm{ng} / \mathrm{ml} \mathrm{HB-EGF}$ (cat. no. 259-HE-050; R\&D Systems, Inc.) added in the basal chamber under M1 cells and $3 \mu \mathrm{g} / \mathrm{ml}$ anti-HB-EGF (cat. no. AF-259-NA; R\&D Systems, Inc.) under M2 cells.

Sample collection. The clinical tissue samples used in this study were obtained from the Tumor Hospital of Harbin Medical University (Harbin, China). A total of 52 cases (39 males and 13 females; mean age, 59 years) of histopathological diagnosis were collected from June 2016 to November 2018. Matched tumor and normal tissue specimens were obtained from the 52 patients undergoing surgery for HNSCC. Specimens at the time of surgery were obtained in accordance with the World Medical Association Declaration of Helsinki ethical guidelines and patients provided written informed consent. Ethical approval was granted by the Ethics Review Committee of Harbin Medical University. All collected specimens were partially embedded in paraffin, partially frozen by tissue embedding agent (Tissue Freezing Medium; Sakura Finetek Japan Co., Ltd.), and the remaining was kept in liquid nitrogen for subsequent detection.

In situ hybridization (ISH). HPV DNA-based ISH was performed using the high risk -HPV 50 probe (Tianjin Institute of Industrial Biotechnology), recognizing high risk-HPV types 16 and 18, in accordance with the manufacturer's instructions. The slides were examined by a microscope with a digital analysis system, including a BX53 Upright microscope (Olympus Corporation) connected to an Olympus DP74 camera (Olympus Corporation) that was linked to a computer with the image capture software.

RNA scope. The cases of p16 IHC and high risk-HPV DNA ISH-positive were further tested by ISH for HPV E6/E7 mRNA, which was performed by hand using the RNAscope 2.5 HD RNAscope (Advanced Cell Diagnostics) in accordance with the manufacturer's instructions.

Irradiation. The fresh clinical tissue was quickly cleaned of blood stains with PBS under sterile conditions. Then, the tissue was cut into two parts, the control group (0 Gy) and radiation group (6 Gy), which were marked. The two groups were immediately transferred and cultured in 10\% RPMI-1640 medium, following which the radiation group ( $6 \mathrm{~Gy}$ ) received irradiation ( $2 \mathrm{~Gy}$ for $3 \mathrm{~min}$ ) in the lab using the Rad Source RS 2000 small animal irradiator (Rad Source Technologies). The optimal dose of X-ray irradiation was $6 \mathrm{~Gy}$, based on preliminary experiments that we performed as well as literature research. Then, the specimens were cultured at $37^{\circ} \mathrm{C}$, in a humidified incubator for $24 \mathrm{~h}$. After $24 \mathrm{~h}$, the tissues were rinsed with fresh PBS, dried with filter paper and rapidly frozen in Optimal Cutting Temperature compound (Sakura Finetek Japan Co., Ltd.) with liquid nitrogen. The tissues were cut into slices with a thickness of $3-5 \mu \mathrm{m}$ and stored at $-80^{\circ} \mathrm{C}$ in sealed slide boxes.

Immunofluorescence staining. Cells and frozen slices were first exposed to radiation, then they were soaked in $4 \%$ paraformaldehyde for $15 \mathrm{~min}$ at room temperature, in $0.5 \%$ Triton X-100 for 5 min, and blocked in 5\% BSA (Beijing Solarbio Science $\&$ Technology Co., Ltd.) for $30 \mathrm{~min}$. Samples were incubated with the $\gamma \mathrm{H} 2 \mathrm{~A}$ histone family member X (H2AX) mouse monoclonal antibody (product code ab26350; Abcam) at 1:500 dilution, primary rabbit polyclonal antibodies against RAD51 recombinase (RAD51; product code ab133534; Abcam) at 1:200, and phosphorylated (p)-DNA-dependent protein kinase (DNA-PK; phosphor S2056; product code ab124918; Abcam) at 1:200 and EGFR inhibitor gefitinib (ZD1839; cat. no. HY-50895; MedChemExpress) overnight at $4^{\circ} \mathrm{C}$. After 
samples were washed three times with PBS (Shanghai Bio-Tech Co., Ltd.), they were incubated for $1 \mathrm{~h}$ at room temperature with the following secondary antibodies (dilution 1:200): Goat anti-mouse IgG (cat. no. BA1126; Wuhan Boster Biological Technology, Ltd.) and Alexa Fuor 488 goat anti-rabbit IgG (H+L) (cat. no. A0423; Beyotime Biotechnology). Samples were then counterstained with DAPI (Beijing Solarbio Science $\&$ Technology Co., Ltd.) to observe the nuclei. The area where the green fluorescence emitted by the $\gamma$-H2AX foci overlapped with the blue-emitting DAPI-stained nucleus was considered to be a radiosensitivity predictor of HNSCC. The proportion of the $\gamma$-H2AX epidemic area to the blue-fluorescent DAPI-stained nuclei was used as the $\mathrm{y}$-axis, and the t-test method was used to calculate the statistical difference. The control group was untreated HNSCC tissue. The green fluorescent $\gamma$-H2AX overlapping with the blue-emitting DAPI-stained nuclei was used as the baseline number of foci per nucleus. The results were visualized by an Olympus BX53 Upright light microscope using a magnification of $x 400, \gamma$-H2AX, RAD51 and p-DNA-PK foci were counted using ImageJ v1.8.0 software (National Institutes of Health). A total of three fields were analyzed for all analyses of immunostaining.

Hematoxylin and eosin (H\&E) staining and immunohistochemistry (IHC). Tumor and normal tissues were fixed in formalin for $2 \mathrm{~h}$ at room temperature. Then, they were processed and embedded in paraffin, and $3 \mu \mathrm{m}$-thick sections were cut and baked at $60^{\circ} \mathrm{C}$ for $30 \mathrm{~min}$. Sections were deparaffinized in three changes of xylene (Tianjin FUYU Fine chemicals Co., Ltd.) for $5 \mathrm{~min}$ each and were then rehydrated using graded concentrations of alcohol. Sections were stained with H\&E (Beijing Solarbio Science \& Technology Co., Ltd.) for $2 \mathrm{~min}$ at room temperature and then sealed with neutral gelatin (item no. G8590; Solarbio Life Sciences).

The expression of p16 (cat. no. TA500036; OriGene Technologies, Inc.), inducible nitric oxide synthase (iNOS; 1:100; product code ab53769; Abcam), CD163 (1:100; cat. no. ab87099; Abcam) and EGFR (1:100; product code ab52894; Abcam) in tumor and normal tissues was determined by IHC analysis. Antigen retrieval was performed using a pressure cooker with $10 \mathrm{mM}$ citrate buffer ( $\mathrm{pH} \mathrm{6.0;}$ Beijing Solarbio Science \& Technology Co., Ltd.), and endogenous peroxidase activity was blocked for $30 \mathrm{~min}$ at room temperature by incubation in $3 \%$ hydrogen peroxide solution (Guangzhou Jiangshun Chemical Technology Co., Ltd.). The slides were incubated with antibodies against p16, iNOS, CD163 and EGFR overnight at $4^{\circ} \mathrm{C}$. The slides were washed with buffer and were incubated with goat anti-mouse/rabbit IgG (cat. no. TA130015; OriGene Technologies, Inc.) for $60 \mathrm{~min}$ at room temperature. ImageJ v1.8.0 software was used to analyze digital images. A total of three fields were analyzed for all analyses of IHC.

Immunofluorescence staining detection. Extracted proteins from HPV-positive and HPV-negative tumor tissues were used for analysis. Conditioned medium was aliquoted and stored frozen $\left(-80^{\circ} \mathrm{C}\right)$ for subsequent analysis. A total of four cytokines [for IL-10, tumor necrosis factor (TNF)- $\alpha$, HB-EGF and EGF] were detected by bead-based immunoassays with multi-cytokine detection kit (Human Magnetic Luminex
Performance Assay Base Kit; cat. no. LHSCM000; R\&D Systems, Inc.) following the manufacturer's guidelines.

Western blotting. Cell lysates were obtained $24 \mathrm{~h}$ after irradiation with RIPA lysis buffer (cat. no. P0013B; Beyotime Institute of Biotechnology) and protease and phosphatase inhibitors (PMSF; cat. no. 90100; Solarbio Life Sciences). Protein concentrations were determined by a BCA kit (Beyotime Institute of Biotechnology). Equal proteins $(10 \mathrm{mg}$ ) were subjected to electrophoresis at $180 \mathrm{~V}$ via $10 \%$ SDS-PAGE and were electrotransferred onto polyvinylidene fluoride membranes (Bio-Rad Laboratories, Inc.) at $4^{\circ} \mathrm{C}$ and $20 \mathrm{~V}$ for $3 \mathrm{~h}$. Membranes were blocked with 5\% BSA overnight at $4^{\circ} \mathrm{C}$. Subsequently, the membranes were incubated with the following primary antibodies: EGFR $(1: 2,000$; product code ab245362; Abcam); p-EGFR (Tyr1173; 1:2,000; cat. no. 44-794G; Thermo Fisher Scientific, Inc.); and $\beta$-actin (1:2,000; cat. no. TA-09; ZSGB-BIO; OriGene Technologies, Inc.) at $4^{\circ} \mathrm{C}$ overnight. Membranes were rinsed in Tris-buffered saline (Beijing Solarbio Science \& Technology Co., Ltd.) with 0.1\% Tween-20 (Beijing Solarbio Science \& Technology Co., Ltd.), followed by incubation with anti-rabbit or anti-mouse IgG antibody (1:5,000; cat. no. BF03008; BOAOLONG) at room temperature for $1 \mathrm{~h}$. Protein bands were visualized using the BeyoECL Plus kit (cat. no. P0018S; Beyotime Institute of Biotechnology. Finally, the membranes were exposed to a gel imaging system ChemiDox XRS+System (ECL developer; cat. no. 1708265; Bio-Rad Laboratories, Inc.). Gray values of protein blots were calculated using ImageJ v1.8.0 software. $\beta$-actin served as the internal control.

TCGA data analysis. RNA-HTSeq-Counts data on 518 HNSCC tissue samples and the corresponding clinical information were downloaded from TCGA (http://www.tcga. org/). The data with total RNA of which expression were detectable in $>90 \%$ samples, was as described by GENCODE (Release 28). A total of 98 patients that were HPV-positive and 420 patients that were HPV-negative were divided by the HPV status according to the clinical information of the HNSCC project from TCGA. The expression of HB-EGF and EGF in $98 \mathrm{HPV}$-positive and $420 \mathrm{HPV}$-negative patients was compared using Student's t-test.

Statistical analysis. Data are expressed as the means of at least three independent experiments. P-values were analyzed using Student's t-test with a two-tailed method. For comparisons between multiple groups, the P-value analysis used ANOVA with Tukey's multiple comparisons test. Pearson's rank test was used for correlation assessments. $\mathrm{P}<0.05$ was considered to indicate a statistically significant difference. GraphPad Prism 7 software (GraphPad Software, Inc.) was used to analyze data.

\section{Results}

Clinical information. Surgical treatment information was collected from 52 patients with HNSCC. Statistical analysis revealed that patients with HNSCC that were HPV-positive were mostly male and HPV infection was related to smoking and alcohol consumption (Table I). 
The histomorphology of tumor and normal tissues was observed by H\&E staining (Fig. 1A). HPV infection was detected by p16 IHC, HPV DNA ISH and RNAscope (Fig. 1B). All 52 specimens, including $20 \mathrm{HPV}$-positive and 32 HPV-negative, p16 IHC and HPV DNA ISH results were consistent. Then, one additional HPV-positive case was found (no=20) through RNAscope. Finally, 19 HPV-positive cases and $33 \mathrm{HPV}$-negative cases were defined. The information of patients was collected in Table I.

The $\gamma-\mathrm{H} 2 \mathrm{AX}$ foci were regarded as a predictor of radiosensitivity in HNSCC (Fig. 1C). There were no significant differences in the number of $\gamma$-H2AX foci in HPV-positive and HPV-negative tumor tissues that were treated by $0 \mathrm{~Gy}$. However, when the tumor tissues were treated by 6 Gy X-ray, the number of $\gamma$-H2AX foci in HPV-positive tumor tissues was significantly higher than the HPV-negative ones $(\mathrm{P}=0.0048$; Fig. 1D). In summary, the results indicated that HPV-positive HNSCC tissues exhibited higher radiosensitivity.

M2 macrophages are highly infiltrated in HPV-negative $H N S C C$ and released $H B-E G F$. To investigate whether the infiltration of M1 and M2 macrophages varies in different types of HNSCC, IHC was performed with antibodies against iNOS (a marker of M1 macrophages) and against CD163 (a marker of M2 macrophages; Fig. 2A). The results demonstrated that M1 infiltration in HPV-positive tumor tissues was higher compared with HPV-negative tissues ( $\mathrm{P}=0.0024$; Fig. 2B); M2 infiltration was higher in HPV-positive tumor tissues compared with HPV-negative tumor tissues ( $\mathrm{P}=0.0045$; Fig. 2C).

The expression of HB-EGF and EGF was measured in HPV-positive and HPV-negative tumor tissues. The results revealed that the HB-EGF expression was higher than EGF in both HPV-positive and HPV-negative tumor tissues. In addition, the expression of HB-EGF in HPV-negative HNSCC tissues was higher than HPV-positive HNSCC tissues $(\mathrm{P}=0.0332$; Fig. 2D). To further evaluate the expression of HB-EGF and EGF in HPV-positive and HPV-negative HNSCC tissues at the transcriptome level, the data of HPV-positive patients $(n=98)$ and HPV-negative HNSCC patients $(n=420)$ was analyzed from the TCGA database. As anticipated, HB-EGF and EGF were upregulated in HPV-negative HNSCC and downregulated in HPV-positive HNSCC $(\mathrm{P}<0.001)$. Moreover, the expression of HB-EGF was higher than EGF in both HPV-positive and HPV-negative HNSCC (Fig. 2E).

Next, the expression of TNF- $\alpha$ and IL-10 was detected, causing the macrophages to polarize into the M1 or M2 type, respectively, in HPV-positive and HPV-negative HNSCC tissues. The expression of TNF- $\alpha$ was significantly higher in HPV-positive tumor tissues than that in HPV-negative tumor tissues ( $\mathrm{P}=0.0185$; Fig. $2 \mathrm{~F})$, but the expression of IL-10 was lower in HPV-positive tumor tissues ( $\mathrm{P}=0.0402$; Fig. $2 \mathrm{~F}$ ). Then, the supernatant was collected from cultured M1 and M2 macrophages and the expression of HB-EGF and EGF was assessed. In the supernatant from both types of macrophages, there was a higher expression level of HB-EGF than EGF. Notably, M2 macrophages secreted significantly higher levels of HB-EGF than M1 macrophages ( $\mathrm{P}=0.007$; Fig. 2G).

Based on these results, the infiltration of M2 macrophages was higher in HPV-negative HNSCC tissues. Additionally, M2 macrophages were the main source of HB-EGF.
Table I. Demographic and clinical characteristics in head neck cancer.

\begin{tabular}{lrrrr}
\hline Characteristics & $\begin{array}{c}\text { Total } \\
\text { no. }\end{array}$ & $\begin{array}{c}\mathrm{HPV}^{+} \\
\mathrm{n}=19\end{array}$ & $\begin{array}{c}\mathrm{HPV}^{-} \\
\mathrm{n}=33\end{array}$ & P-value \\
\hline Sex & & & & 0.937 \\
$\quad$ Male & 39 & 15 & 24 & \\
Female & 13 & 4 & 9 & \\
Age & & & & 0.770 \\
$<50$ & 5 & 1 & 4 & \\
$>$ 50 & 47 & 28 & 19 & \\
Smoking & & & & 0.007 \\
Never & 15 & 3 & 12 & \\
$<20$ years & 5 & 5 & 0 & \\
$>$ 20 years & 32 & 7 & 25 & \\
Alcohol & & & & 0.006 \\
Never & 36 & 11 & 27 & \\
$<20$ years & 2 & 1 & 1 & \\
$>$ 20 years & 14 & 8 & 6 & \\
Node status & & & & \\
N0-N1 & 50 & 13 & 37 & \\
N2-N3 & 2 & 1 & 1 & \\
Differentation & & & & \\
Well & 21 & 10 & 11 & \\
Moderate & 18 & 7 & 12 & \\
Poor & 13 & 1 & & \\
\hline
\end{tabular}

HPV, human papilloma virus.

$M 2$ macrophages reduce the radiosensitivity of $H P V$-negative $H N S C C$ by releasing $H B-E G F$. The HB-EGF expression against the $\gamma$-H2AX foci within $24 \mathrm{~h}$ after $6 \mathrm{~Gy}$ irradiation is presented in Fig. 3A, indicating a significant negative correlation between the expression of HB-EGF and $\gamma$-H2AX foci in HNSCC tissues ( $\mathrm{r}=0 .-8131$ ).

To determine the role of HB-EGF in radiation responses, the radiosensitivity of the SCC90 cell line (HPV-positive) and the CAL27 cell line (HPV-negative) was detected with or without HB-EGF (Fig. 3B). In the SCC90 cell line, the radiation sensitivity was reduced when HB-EGF was added $(\mathrm{P}=0.0011)$, and the results of CAL27 were consistent ( $\mathrm{P}=0.0037$; Fig. 3C).

To establish the M2 macrophage-HB-EGF-radiosensitivity model, CAL27 cells co-cultured with polarized macrophages for $24 \mathrm{~h}$ were subjected to a radiosensitivity assay (Fig. 3D). The CAL27 cells co-cultured with M1 macrophages exhibited higher radiosensitivity compared with CAL27 cells co-cultured with M2 macrophages ( $\mathrm{P}=0.0012$; Fig. 3E). Notably, M2 macrophages co-cultured with CAL27 cells exhibited higher sensitivity to radiation therapy when the anti-HBEGF-neutralizing antibody was added $(\mathrm{P}=0.0127$; Fig. 3F). The CAL27 cells co-cultured with M1 macrophages exhibited a significant decrease in radiosensitivity after treatment with HB-EGF $(\mathrm{P}=0.0155$; Fig. 3G). These results supported the model in which M2 macrophages decreased radiosensitivity in HPV-negative HNSCC by secreting HB-EGF. 
A
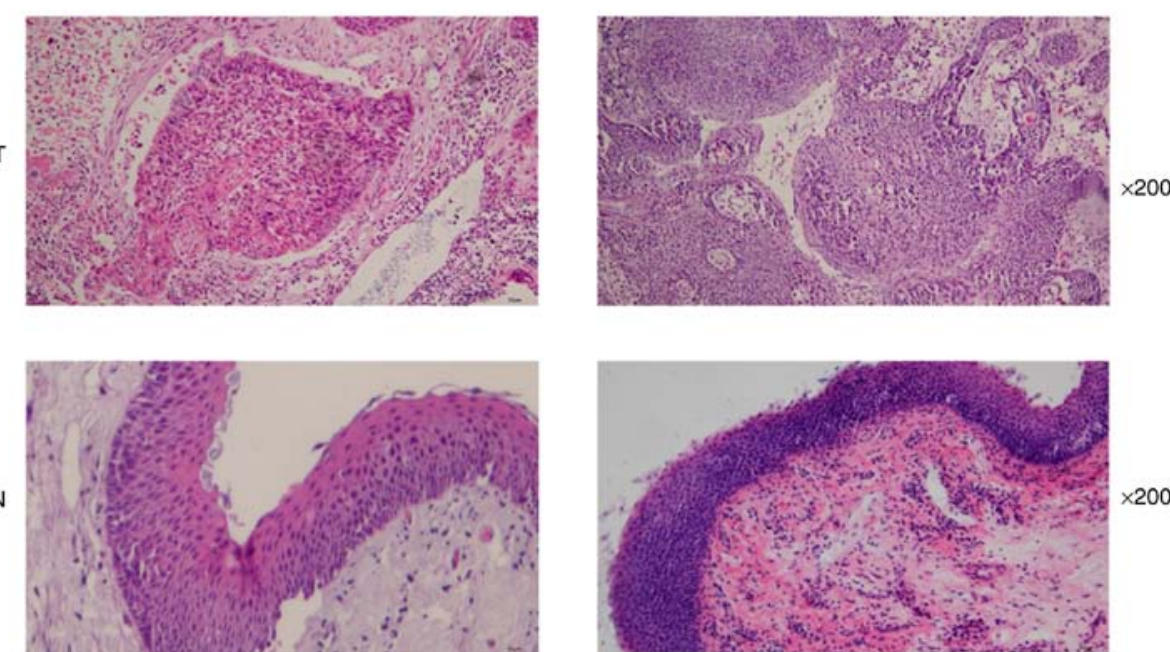

B
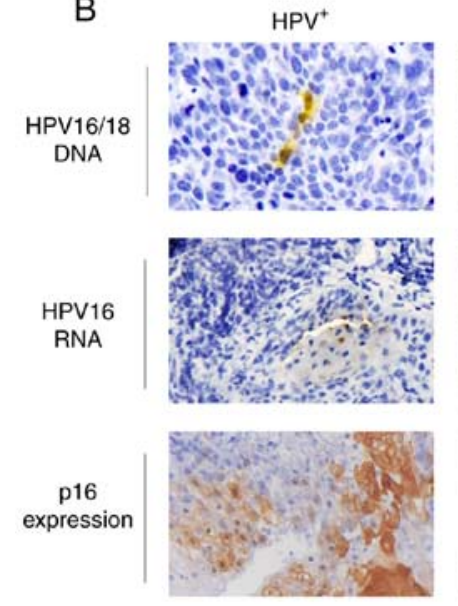

$\mathrm{HPV}^{-}$
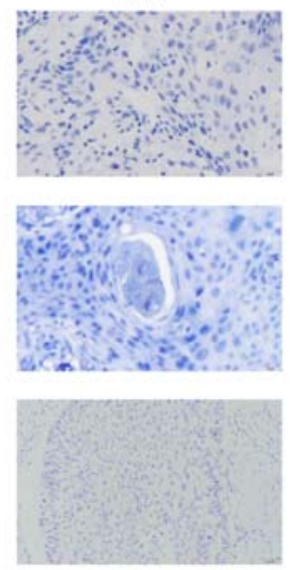

$\mathrm{P}$
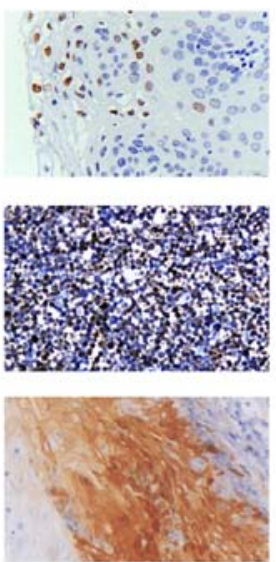

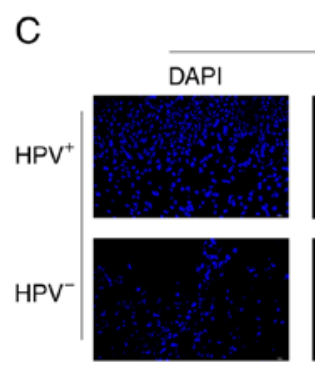

0 Gy
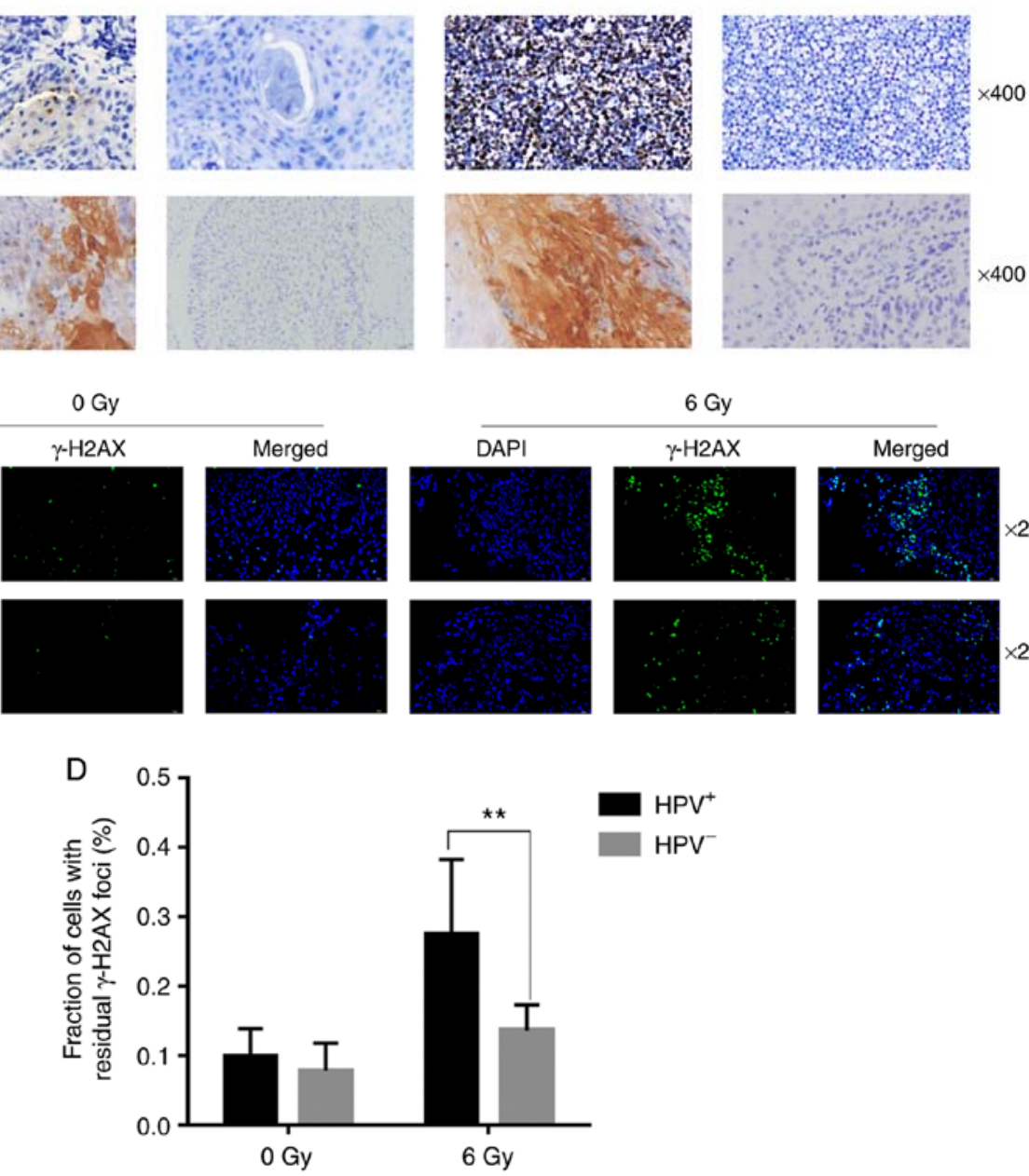

Figure 1. Detection of HPV infection and radiosensitivity in HNSCC tissues. (A) Representative images of H\&E staining using magnification, $\mathrm{x} 200$ (T, HNSCC tissues; N, head and neck normal tissues). (B) Representative images of negative and positive HPV-16/18 detected by DNA ISH. Negative and positive HPV16 RNA ISH detected by RNAscope. Negative and positive p16 detected by 16 IHC using magnification, x200 ( $\mathrm{HPV}^{+}$, HPV-positive tumor tissues; HPV T, $^{-}$ HPV-negative tumor tissues; $\mathrm{HPV}^{+} / \mathrm{HPV}^{-} \mathrm{N}$, head and neck normal tissues; P, positive control-cervical cancer tissues; $\mathrm{N}$, negative control-glioma tissues). (C) Representative images of the $\gamma$-H2AX staining of frozen HNSCC sections used to evaluate the extent of radiosensitivity; magnification, x200 (green, $\gamma$-H2AX staining; blue, DAPI). (D) Number of the $\gamma$-H2AX foci per cell; HPV-positive cases $(n=19)$, HPV-negative cases $(n=33)$. Results are presented as the mean $\pm \mathrm{SD} .{ }^{* * *} \mathrm{P}<0.01$. HPV, human papilloma virus; HNSCC, head and neck squamous cell carcinoma; H\&E, hematoxylin and eosin; ISH, in situ hybridization; IHC, immunohistochemistry; $\gamma$-H2AX, $\gamma$ H2A histone family member X. 
A
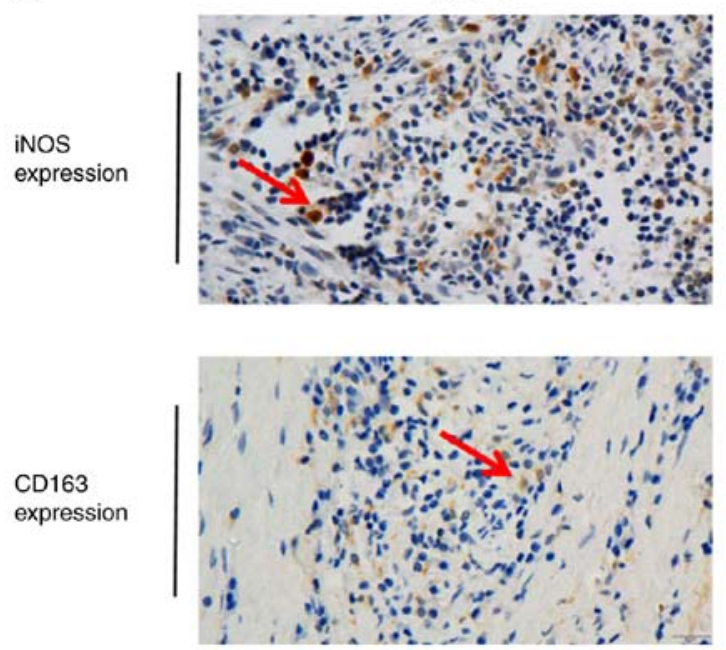

B

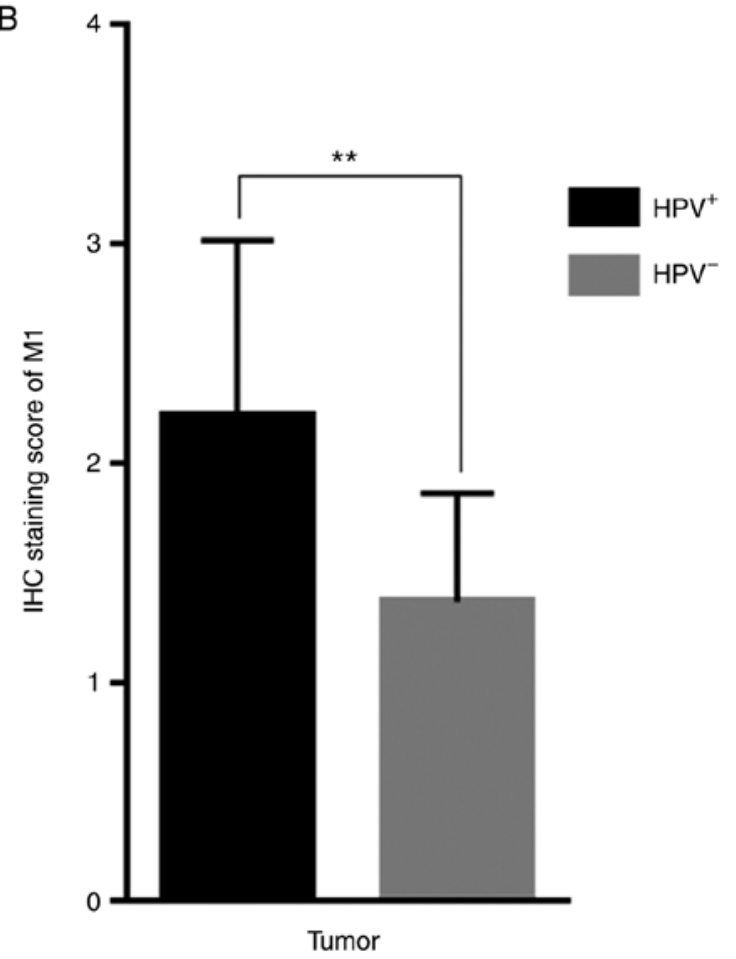

D

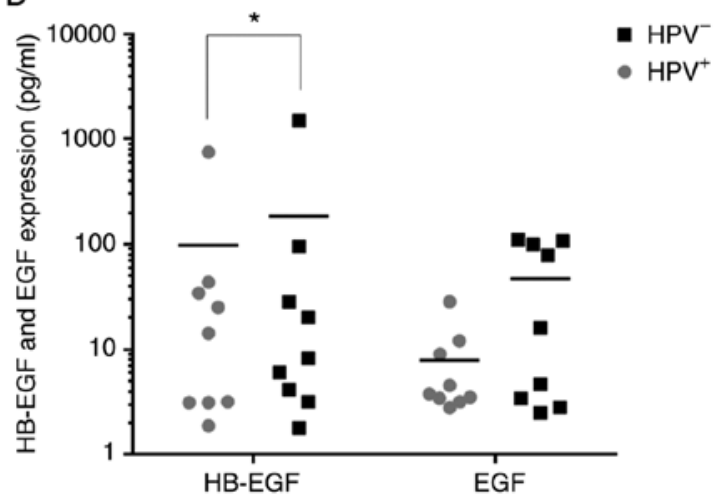

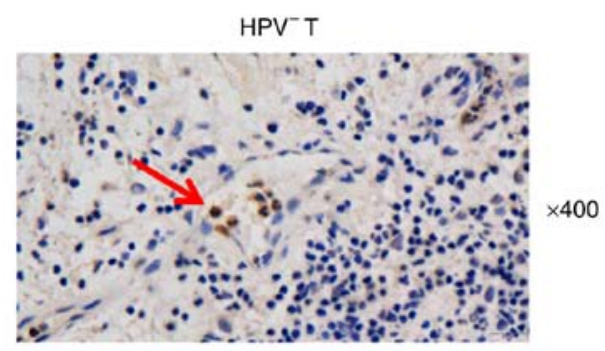

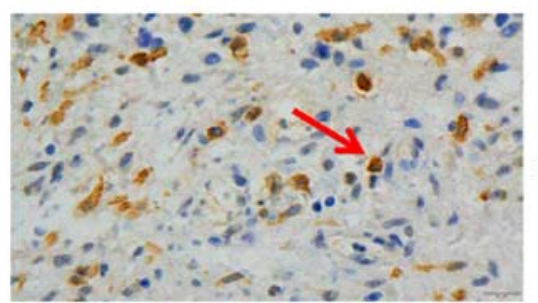

$\times 400$

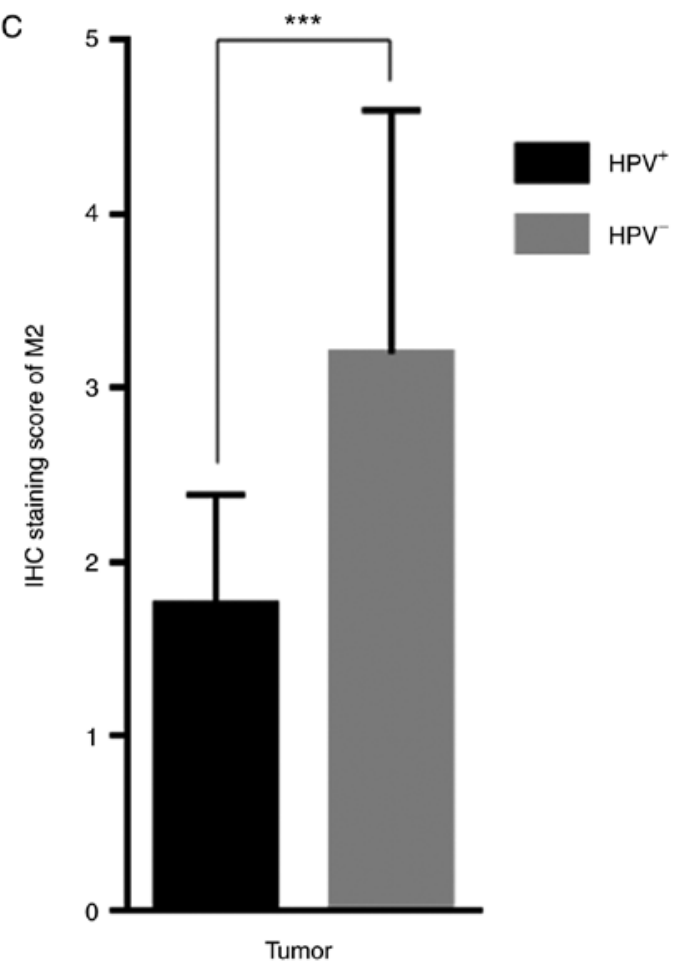

$\mathrm{E}$

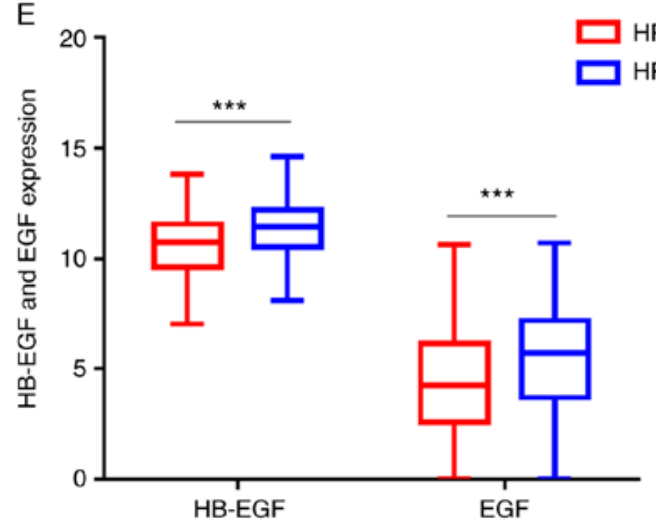

Figure 2. Infiltration of M2 macrophages is high in HPV-negative HNSCC and M2 macrophages release HB-EGF. (A) IHC staining of HNSCC tumor and normal tissues using anti-iNOS and anti-CD163 antibodies; magnification, $\mathrm{x} 400$ ( $\mathrm{HPV}^{+} \mathrm{T}$, HPV-positive tumor tissues; HPV $\mathrm{T}$, HPV-negative tumor tissues). Arrows indicate dot-like hybridization signals in tumor cell nuclei. (B and C) iNOS and CD163 IHC score. HPV-positive cases (n=19), HPV-negative cases $(\mathrm{n}=33) .{ }^{* *} \mathrm{P}<0.01,{ }^{* * *} \mathrm{P}<0.001$. (D) Expression profiles of HB-EGF and EGF from 9 HNSCC clinical samples were assessed by multiple-cytokine detection. ${ }^{*} \mathrm{P}<0.05$. (E) Box plot showing differences in the expression of HB-EGF and EGF in HPV-positive ( $\left.\mathrm{n}=98\right)$ and HPV-negative (n=420) HNSCC in the TCGA database. ${ }^{* * *} \mathrm{P}<0.001$. 

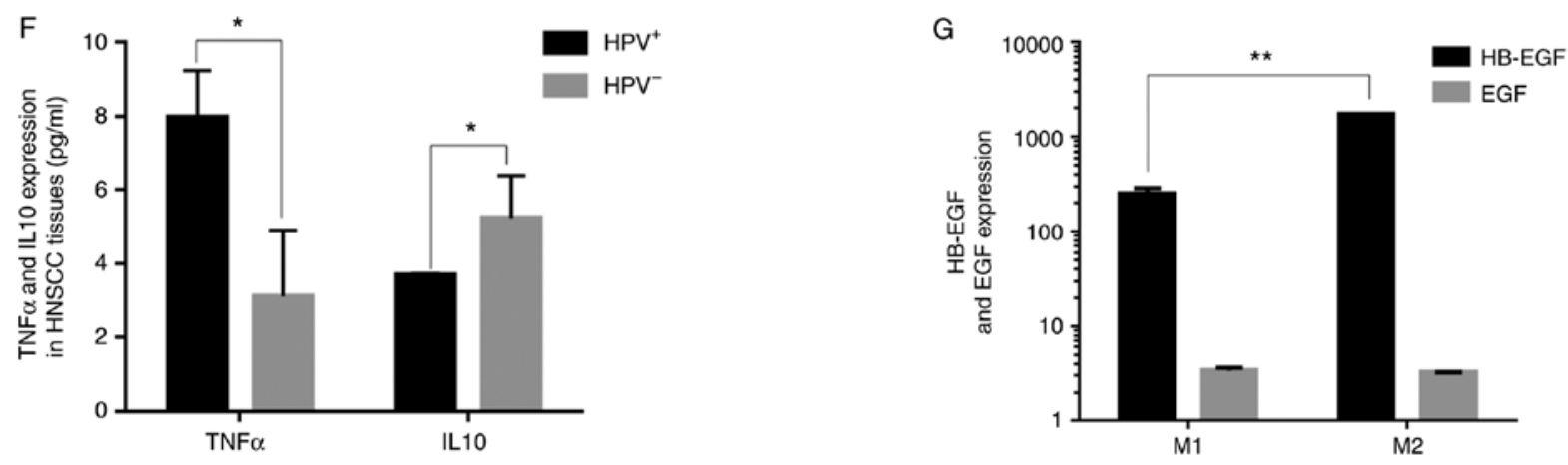

Figure 2. Continued. Infiltration of M2 macrophages is high in HPV-negative HNSCC and M2 macrophages release HB-EGF. (F) Expression profiles of TNF- $\alpha$ and IL-10 from 12 HNSCC clinical samples were detected by the multiple-cytokine detection method. (G) Expression profiles of HB-EGF and EGF from the supernatants of M1 and M2 were detected by the multiple-cytokine detection method. Results are presented as the mean $\pm \mathrm{SD}$. ${ }^{*} \mathrm{P}<0.05,{ }^{* *} \mathrm{P}<0.01$. HPV, human papilloma virus; HNSCC, head and neck squamous cell carcinoma; HB-EGF, heparin-binding epidermal growth factor; iNOS, inducible nitric oxide synthase; EGF, epidermal growth factor; TCGA, The Cancer Genome Atlas; TNF, tumor necrosis factor; IL, interleukin; IHC, immunohistochemistry.

Inhibition of EGFR promotes the non-homologous endjoining (NHEJ) repair pathway. To further determine the downstream pathway by which HB-EGF decreased radiosensitivity, the phosphorylation of EGFR was detected in CAL27 cells and CAL27 cells treated with HB-EGF. Western blotting experiments using phosphospecific antibodies to recognize the activated EGFR revealed that when only HB-EGF was added, the p-EGFR level was significantly increased in CAL27 cells. However, under 4 Gy irradiation, p-EGFR expression was increased in the HB-EGF group compared with the non-HB-EGF group (EGFR, $\mathrm{P}=0.0049$; $\mathrm{p}$-EGFR, $\mathrm{P}=0.0044$; Fig. 4A and B, respectively).

To investigate which pathway of the two principal DNA double-strand break (DSB) repair pathways [NHEJ and homologous recombination (HR)] is the main pathway activated by the combination of HB-EGF and EGFR, RAD51 (a specific marker for HR) and p-DNA-PK (a specific marker for NHEJ) expression levels were detected $5 \mathrm{~h}$ after CAL27 cells treated with HB-EGF and the EGFR inhibitor were exposed to radiation (Fig. 4C and D). Immunofluorescence analysis showed that the number of p-DNA-PK foci of CAL27 cells treated with HB-EGF alone was significantly higher than that of the untreated group $(\mathrm{P}=0.04531)$, and the number of p-DNA-PK foci was significantly reduced after the addition of EGFR inhibitor $(\mathrm{P}=0.04319)$ indicating that HB-EGF activated EGFR and then promoted the induction of NHEJ (Fig. 4E-a). In contrast, there was no significant difference in the number of RAD51 lesions after the addition of HB-EGF $(\mathrm{P}=0.07562)$, and there was no significant difference after the addition of EGFR inhibitor ( $\mathrm{P}=0.08866$; Fig. 4E-b). These findings indicated that NHEJ was the main pathway activated by the interaction of HB-EGF and EGFR.

To eliminate the effect of HPV infection on EGFR activation, the expression of EGFR was detected in HPV-positive and HPV-negative tumors, which was then compared to normal tumors. In both HPV-positive and HPV-negative HNSCC, the expression of EGFR was higher in tumor tissues. Notably, the expression of EGFR was significantly higher in HPV-negative HNSCC than HPV-positive $(\mathrm{P}=0.0492$; Fig. $4 \mathrm{~F}$ and $\mathrm{G})$. To confirm the differences in EGFR expression, data from the TCGA database were analyzed, which revealed the upregulation of EGFR in HPV-negative HNSCC and downregulation in HPV-positive HNSCC $(\mathrm{P}=0.0312$; Fig. $4 \mathrm{H})$.

\section{Discussion}

The present study revealed that HPV-positive HNSCC tissues were more sensitive to radiotherapy (19). In HPV-negative HNSCC tissues, there was increased M2 macrophage infiltration compared with HPV-positive HNSCC tissues, and these macrophages released HB-EGF to induce radiotherapy resistance. This was related to the interaction between HB-EGF and EGFR, and the induction of the NHEJ pathway.

HNSCC can be divided into HPV-positive and HPV-negative (20). In recent years, a number of studies have revealed that patients with HPV-positive HNSCC have increased sensitivity to radiotherapy and chemotherapy, and they exhibited improved prognosis rates $(21,22)$. However, patients with HPV-negative HNSCC exhibited higher levels of resistance to radiotherapy and chemotherapy, which causes tumor recurrence and poor prognosis rates (23). Prevc et al (24) revealed that immunodeficient mice with HPV-positive tumors had a 30\% higher radiosensitivity level. Arenz et al (25) demonstrated that the increased radiosensitivity of HPV-positive HNSCC cell lines was a result of cell cycle dysregulation and the induction of apoptosis, however the underlying mechanism was unclear. Since in HPV-positive cell lines such as SCC090, HPV appears in multiple copies in a free or integrated state, and each cell has multiple oncogenes, such as E6 and E7, it is therefore difficult to achieve full gene knockout of the HPV virus (26). In addition, some research results revealed that the knockout of HPV16-E7 by the CRISPR/CAS system induced apoptosis and growth inhibition in HPV16-positive cells $(27,28)$. In order to avoid the effect of gene knockout on cell changes in subsequent experiments, our experiments did not use the HPV-positive HNSCC cell line combined with knockout technology, but instead it used the HPV negative HNSCC cell line binding inhibitor for mechanism research.

Recently, studies have revealed that the tumor immune microenvironment plays an important role in the treatment of HNSCC $(29,30)$. Compared with HPV-negative tumors, an increased number of immune cells infiltrate HPV-positive tumors, among which TAMs play an important role (13). 

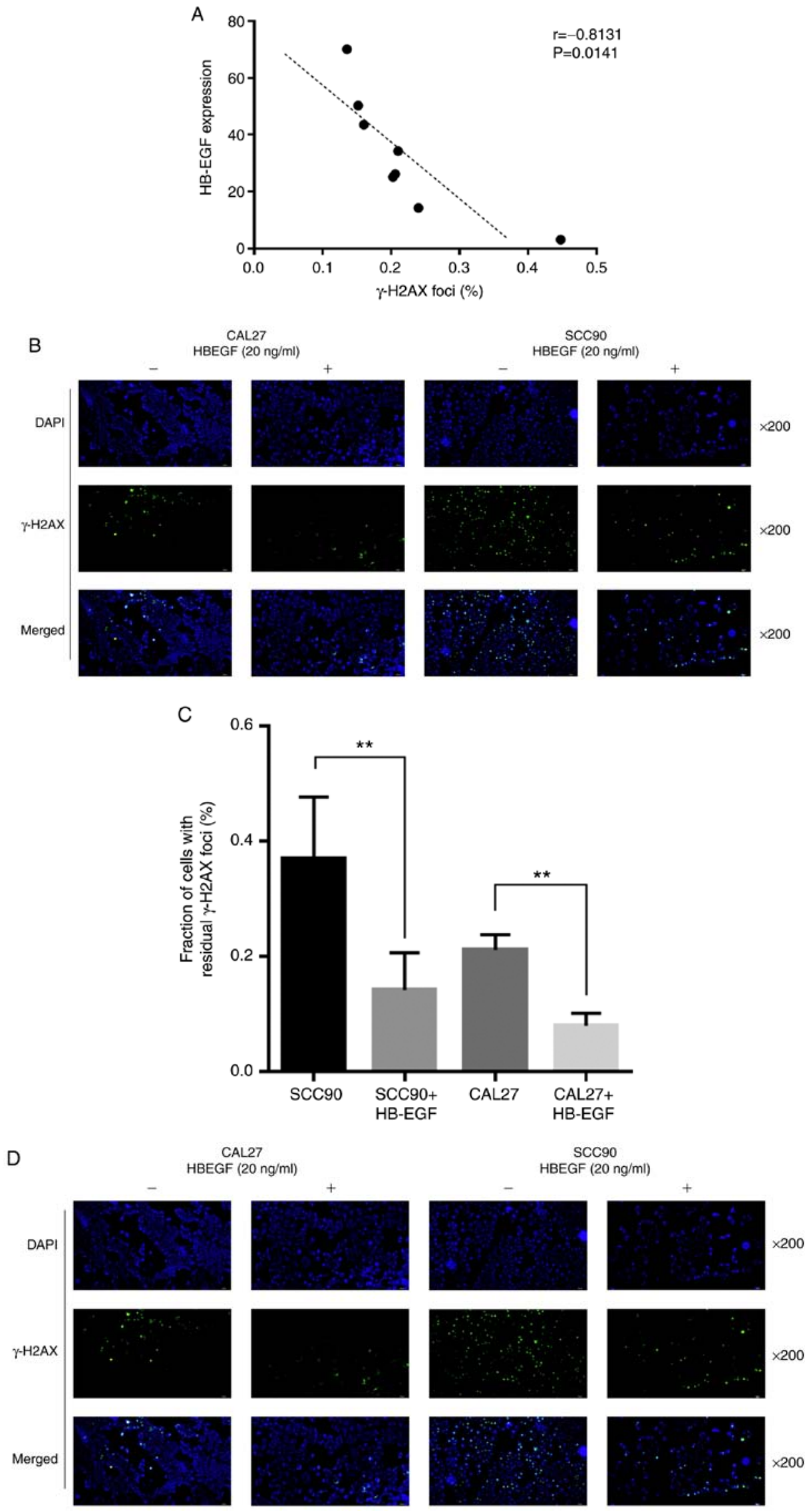

Figure 3. M2 macrophages reduce the radiosensitivity of HPV-negative HNSCC by releasing HB-EGF. (A) Correlation analyses between the fraction of the $\gamma$-H2AX foci and the expression of HB-EGF. (B) Representative images of fluorescent staining. Immunofluorescence analyses revealing the expression of $\gamma$-H2AX foci in CAL27 and SCC90 cells with or without HB-EGF $(20 \mathrm{ng} / \mathrm{ml}) 5 \mathrm{~h}$ after 4 Gy radiation; magnification, $\mathrm{x} 200$ (green, $\gamma$-H2AX staining; blue, DAPI). (C) Fraction of CAL27 and SCC90 cells with the residual $\gamma$-H2AX foci (\%) with or without HB-EGF (50 ng/ml). ${ }^{* *} \mathrm{P}<0.01$. (D) Immunofluorescence analyses of the $\gamma$-H2AX foci of M2 macrophages co-cultured with CAL27 or SCC90 and anti-HB-EGF antibody ( $\mu \mu \mathrm{g} / \mathrm{ml})$, and M1 macrophages co-cultured with CAL27 or SCC90 and HB-EGF (20 ng/ml); magnification x200 (green, $\gamma$-H2AX staining; blue, DAPI). 

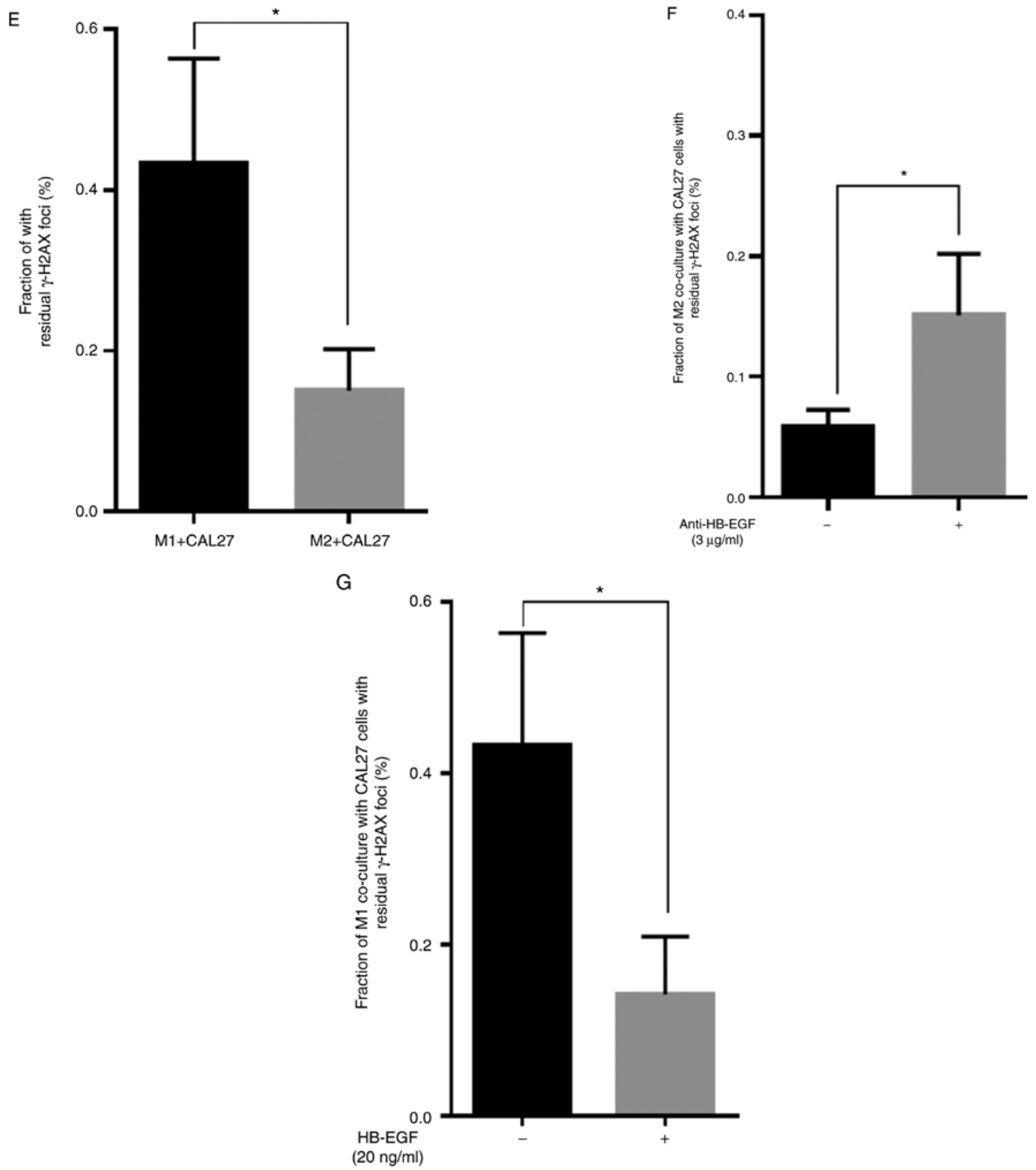

Figure 3. Continued. M2 macrophages reduce the radiosensitivity of HPV-negative HNSCC by releasing HB-EGF. (E) Number of the $\gamma$-H2AX foci of M1 and M2 macrophages co-cultured with CAL27. (F) Number of the $\gamma$-H2AX foci of M2 macrophages co-cultured with CAL27 cells with anti-HBEGF-neutralizing antibody. (G) Number of the $\gamma$-H2AX foci of M1 macrophages co-cultured with CAL27 cells with HB-EGF. Results are presented as mean \pm SD. "P $<0.05$. HPV, human papilloma virus; HNSCC, head and neck squamous cell carcinoma; HB-EGF, heparin-binding epidermal growth factor; $\gamma$-H2AX, $\gamma \mathrm{H} 2 \mathrm{~A}$ histone family member $\mathrm{X}$.

TAMs are mainly divided into M1 macrophages that secrete pro-inflammatory cytokines, such as TNF- $\alpha$ or IL-1 $\beta$, and M2 macrophages that secrete inhibitory cytokines, such as IL-10 or TGF- $\beta$ (31). Most researchers have hypothesized that the infiltration of M2 macrophages leads to poor prognosis in patients with HPV-negative HNSCC, and data from TCGA revealed that high infiltration of $\mathrm{M} 2$ macrophages decreased the survival rates of patients with $\operatorname{HNSCC}(32,33)$. Consistent with this statement, Rades et al (34) revealed that radioresistance led to poor prognosis in HNSCC. In the present study, IHC with the M1 and M2 surface markers iNOS and CD163 was used to detect M1 and M2 in patient tissues. Higher levels of M2 macrophage infiltration was revealed in HPV-negative tissues, and the radiosensitivity of HPV-negative HNSCC tissues was lower compared with positive tissues. Therefore, it is proposed that the infiltration of M2 macrophages may be related to radioresistance of HPV-negative HNSCC.

In the present study, it was demonstrated that M2 macrophages released higher levels of HB-EGF compared with M1 macrophages. The expression of HB-EGF was significantly higher in HPV-negative tissues. HB-EGF was first discovered in 1990, and macrophages are the main source of HB-EGF (17). It has been reported that HB-EGF alleviates DNA damage responses in tumor cells and enhances radioresistance in the 
A

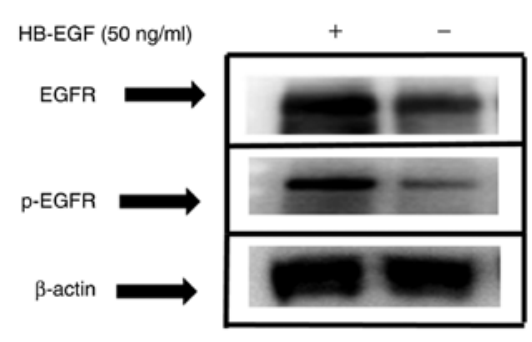

C
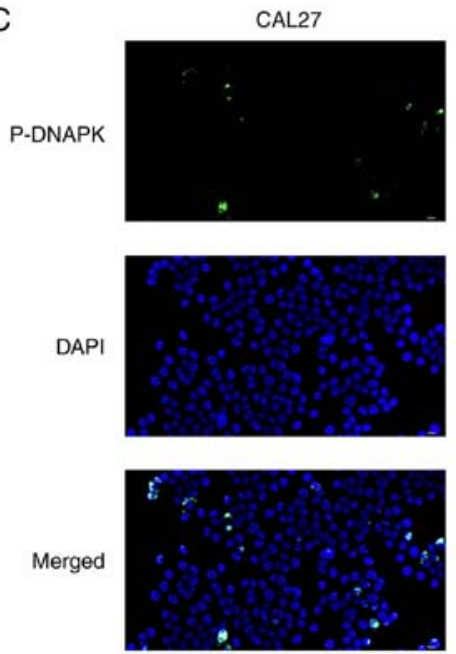

D
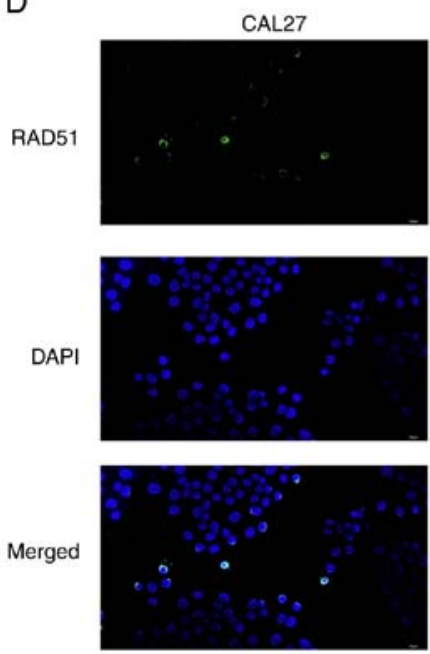

B

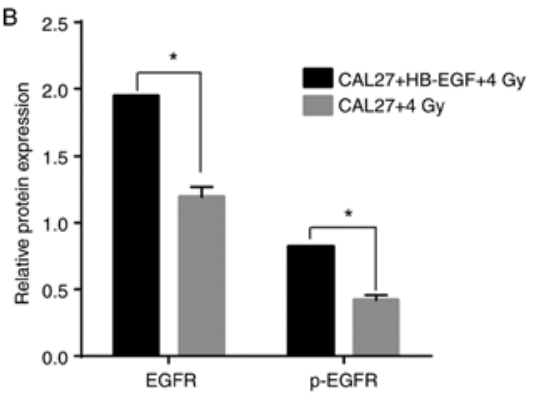

CAL27+HB-EGF
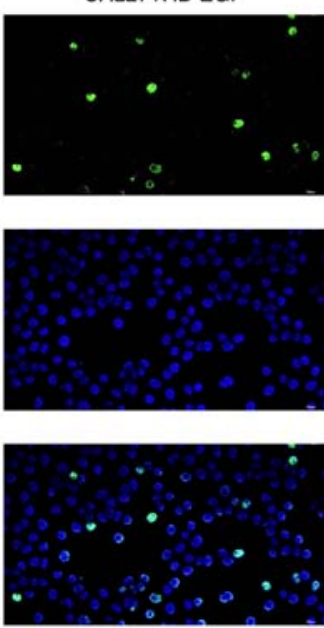

CAL27+HB-EGF
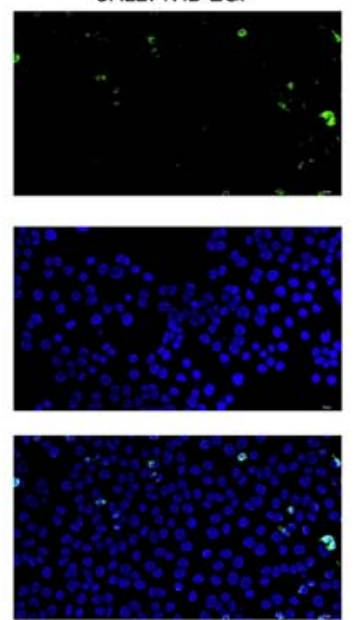
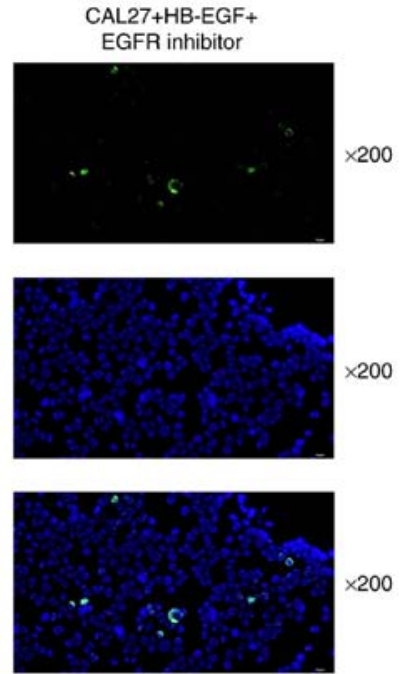

CAL27+HB-EGF+ EGFR inhibitor
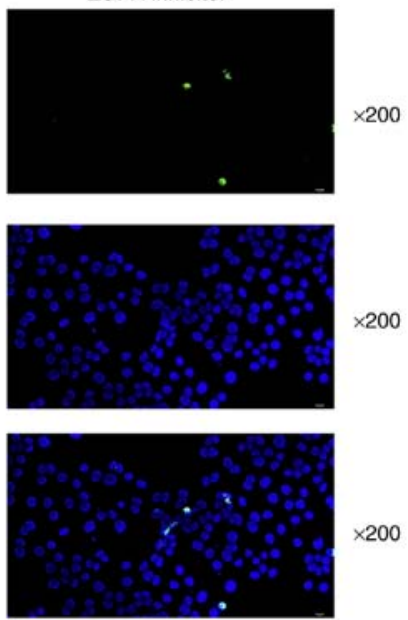

Figure 4. In CAL27 cells, inhibition of EGFR by small molecule inhibitor results in reduced NHEJ repair capacity. (A) CAL27 and CAL27 with HB-EGF $(50 \mathrm{ng} / \mathrm{ml})$ were assessed by the western blot analysis with anti-EGFR and anti-p-EGFR antibodies. (B) EGFR and EGFR phosphorylation expression in CAL27 cells with or without HB-EGF after 4 Gy radiation. NC, CAL27 cells. (C) Representative images of the number of the p-DNA-PK foci per cell in $5 \mathrm{~h}$ after irradiation (4 Gy) in CAL27 cells with or without the EGFR inhibitor; magnification, x200 (green, p-DNA-PK staining; blue, DAPI). (D) Representative images of the number of the RAD51 foci per cell in $5 \mathrm{~h}$ after irradiation (4 Gy) in CAL27 cells with or without the EGFR inhibitor; magnification x200 (green, p-DNA-PK staining; blue, DAPI). ${ }^{*} \mathrm{P}<0.05$.

intestinal canal $(18,35)$. Previous studies have demonstrated that M2 macrophages induced the invasion and migration of myxoid liposarcoma cells and induced the proliferation of ovarian cancer cells by releasing HB-EGF (36-39). The present results also revealed the negative association between the expression of HB-EGF and radiosensitivity. HPV-positive and -negative cell lines SCC090 and CAL27 were selected, and cytological methods were used to co-culture M1 and M2 macrophages with CAL27 cells, and combined HB-EGF inhibitors were used to study whether the secretion of HB-EGF by M2 macrophages decreases the radiation sensitivity of HNSCC cells. Consistent with previous research, it was demonstrated that M2 macrophages played an important role in the development of radioresistance in HPV-negative HNSCC by releasing HB-EGF.

HB-EGF has been revealed to be the ligand with the highest affinity for EGFR in cervical cancer, and HB-EGF could activate EGFR phosphorylation (40). Several previous 

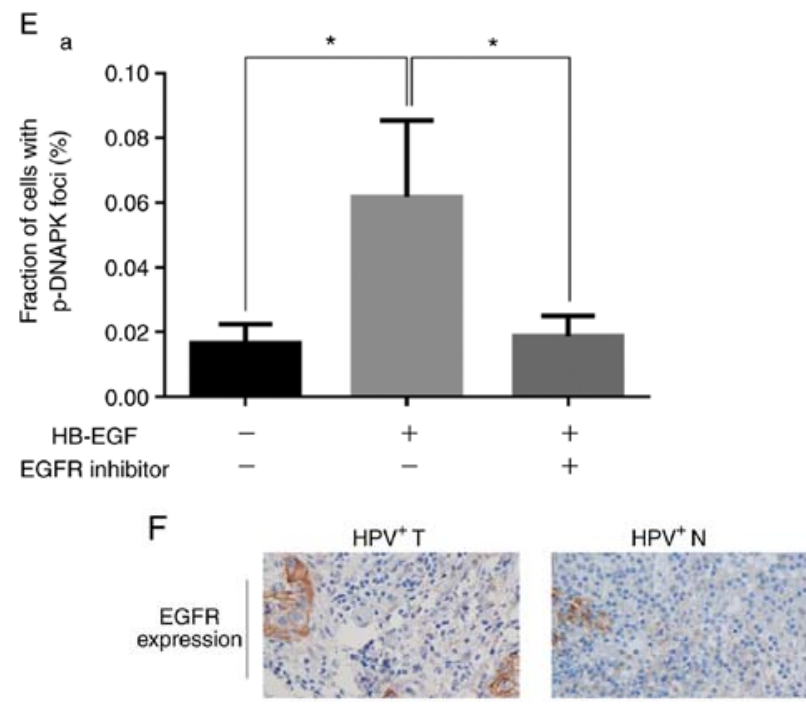

G

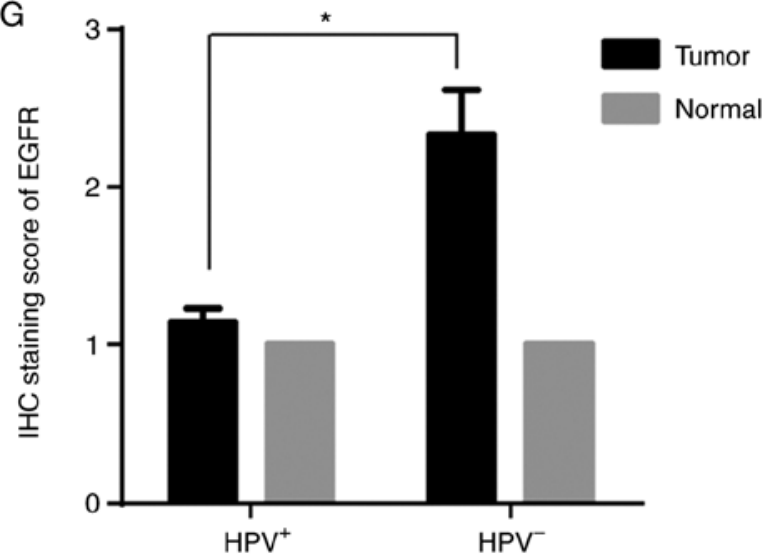

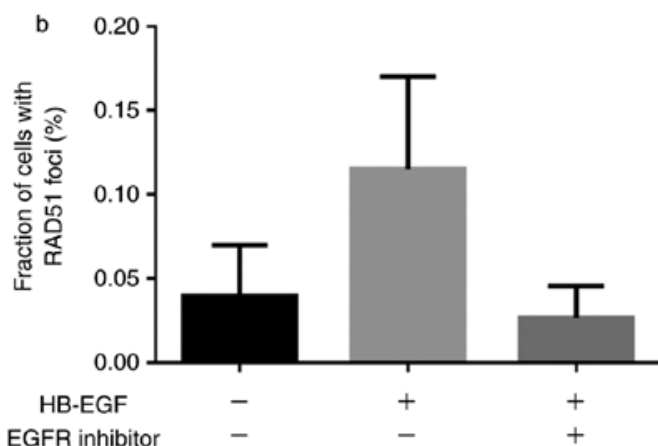

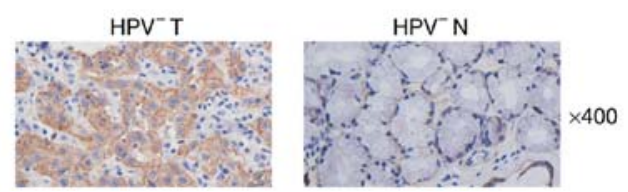

$\mathrm{H}$

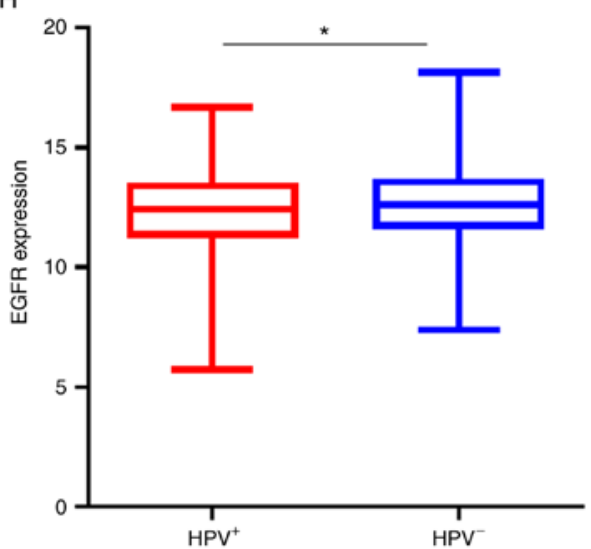

Figure 4. Continued. In CAL27 cells, inhibition of EGFR by small molecule inhibitor results in reduced NHEJ repair capacity. (E-a) Number of p-DNA-PK foci of NHEJ specific markers in CAL27 cells with or without EGFR inhibitors; (E-b) Number of RAD51 foci of HR specific markers in CAL27 cells with or without EGFR inhibitors. (F) IHC analyses of EGFR in cancer and normal tissues; magnification, $\mathrm{x}_{400}$ (HPV ${ }^{+} \mathrm{T}$, HPV-positive tumor tissues; HPV-T, HPV-negative tumor tissues; HPV ${ }^{+} \mathrm{HPV}^{-} \mathrm{N}$, head and neck normal tissues). (G) EGFR IHC score. HPV-positive cancer tissues (n=19), HPV-negative cases $(n=33)$. (H) Expression of EGFR between HPV-positive $(n=98)$ and HPV-negative $(n=420)$ HNSCC in the TCGA database. Results are presented as the mean \pm SD. ${ }^{*} \mathrm{P}<0.05$. HPV, human papilloma virus; HNSCC, head and neck squamous cell carcinoma; EGFR, epidermal growth factor receptor; NHEJ, non-homologous end-joining; HB-EGF, heparin-binding epidermal growth factor; p-, phosphorylated; DNA-PK, DNA-dependent protein kinase; RAD51, RAD51 recombinase; IHC, immunohistochemistry; TCGA, The Cancer Genome Atlas.

studies have revealed that EGFR triggers a downstream signal cascade via autophosphorylation (41-43). In the present study, western blotting, using phosphorylation-specific antibodies to identify activated EGFR, revealed that p-EGFR was significantly increased in CAL27 cells when HB-EGF was added, indicating that EGFR was activated by HB-EGF. However, under 4 Gy irradiation, p-EGFR expression was increased in the HB-EGF group compared with the non-HB-EGF group.

Both classical pathways for DSB repair (NHEJ and HR) are affected by EGFR signaling. A study by Kriegs et al (44) demonstrated that radiation and the activation of EGFR stimulated the MAPK pathway in order to regulate the NHEJ repair pathway, while Toulany et al (45) demonstrated that $\mathrm{PI} 3 \mathrm{~K} / \mathrm{Akt} / \mathrm{mTOR}$ signaling was activated to regulate the HR repair pathway after EGFR was activated. The present results revealed that the NHEJ pathway, but not the HR pathway, was stimulated after EGFR was activated. Regarding DNA damage repair and related pathways, only the EGFR and
NHEJ pathways have been studied, and deeper mechanisms have yet to be explored.

In summary, the present study indicated that M2 macrophages can induce radioresistance of HPV-negative HNSCC by releasing HB-EGF to activate EGFR that can enhance the NHEJ repair pathway. This finding further explores the causes of radiotherapy resistance in patients with HPV-negative HNSCC, indicating that M2 macrophages and HB-EGF are possible regulatory targets for future treatment of HNSCC, and provides new insights into HNSCC radiotherapy.

\section{Acknowledgements}

Not applicable.

\section{Funding}

The present study was supported by the National Natural Science Foundation of China (grant no. 81672670), the 
Heilongjiang Province Outstanding Youth Foundation (grant no. JC2018023), the Graduate Innovation Research Project of Harbin Medical University (grant no. YJSCX2017-8HYB) and the Medical and Health Science and Technology Plan Project of Longgang District, Shenzhen (LGKCYLWS2019000100).

\section{Availability of data and materials}

The datasets used during the present study are available from the corresponding author upon reasonable request.

\section{Authors' contributions}

The study was conceived and designed by EF and LW. JG, PY, JS provided the clinical specimens. EF, TL, XM, XC, LS, HL, FM, SZ, JG, PY, JS and SH conducted the experiments and analyzed data. The manuscript was written by EF, SY and TL. All authors read and approved the final manuscript and agree to be accountable for all aspects of the work in ensuring that questions related to the accuracy or integrity of any part of the work are appropriately investigated and resolved.

\section{Ethics approval and consent to participate}

Ethical approval was granted by the Ethics Review Committee of Harbin Medical University. Specimens at the time of surgery were obtained in accordance with the World Medical Association Declaration of Helsinki ethical guidelines and patients provided written informed consent.

\section{Patient consent for publication}

Not applicable.

\section{Competing interests}

The authors declare that they have no competing interests.

\section{References}

1. Torre LA, Bray F, Siegel RL, Ferlay J, Lortet-Tieulent J and Jemal A: Global cancer statistics, 2012. CA Cancer J Clin 65: 87-108, 2015

2. Park S, Jang WJ and Jeong CH: Nano-biomechanical validation of epithelial-mesenchymal transition in oral squamous cell carcinomas. Biol Pharm Bull 39: 1488-1495, 2016.

3. Solomon B, Young RJ and Rischin D: Head and neck squamous cell carcinoma: Genomics and emerging biomarkers for immunomodulatory cancer treatments. Semin Cancer Biol 52 228-240, 2018

4. Mosca L, Pagano M, Ilisso CP, Cave DD, Desiderio V, Mele L, Caraglia M, Cacciapuoti G and Porcelli M: AdoMet triggers apoptosis in head and neck squamous cancer by inducing ER stress and potentiates cell sensitivity to cisplatin. J Cell Physiol 234: 13277-13291, 2019.

5. Marur S and Forastiere AA: Head and neck squamous cell carcinoma: Update on epidemiology, diagnosis, and treatment. Mayo Clin Proc 91: 386-396, 2016.

6. Ou D, Adam J, Garberis I, Blanchard P, Nguyen F, Levy A, Casiraghi O, Gorphe P, Breuskin I, Janot F, et al: Influence of tumor-associated macrophages and HLA class I expression according to HPV status in head and neck cancer patients receiving chemo/bioradiotherapy. Radiother Oncol 130: 89-96, 2019.

7. Mirghani H, Amen F, Tao Y, Deutsch E and Levy A: Increased radiosensitivity of HPV-positive head and neck cancers: Molecular basis and therapeutic perspectives. Cancer Treat Rev 41: 844-852, 2015.
8. Tian L, Yi X, Dong Z, Xu J, Liang C, Chao Y, Wang Y, Yang $\mathrm{K}$ and Liu Z: Calcium bisphosphonate nanoparticles with chelator-free radiolabeling to deplete tumor-associated macrophages for enhanced cancer radioisotope therapy. ACS Nano 12: 11541-11551, 2018

9. She L, Qin Y, Wang J, Liu C, Zhu G, Li G, Wei M, Chen C, Liu G, Zhang D, et al: Tumor-associated macrophages derived CCL18 promotes metastasis in squamous cell carcinoma of the head and neck. Cancer Cell Int 18: 120, 2018.

10. Barikbin R, Berkhout L, Bolik J, Schmidt-Arras D, Ernst T, Ittrich H, Adam G, Parplys A, Casar C, Krech T, Karimi K, et al: Early heme oxygenase 1 induction delays tumour initiation and enhances DNA damage repair in liver macrophages of Mdr2 ${ }^{-/}$ mice. Sci Rep 8: 16238, 2018

11. Vitale I, Manic G, Coussens LM, Kroemer G and Galluzzi L: Macrophages and metabolism in the tumor microenvironment. Cell Metab 30: 36-50, 2019.

12. Petty AJ and Yang Y: Tumor-associated macrophages: Implications in cancer immunotherapy. Immunotherapy 9: 289-302, 2017.

13. Chen X, Yan B, Lou H, Shen Z, Tong F, Zhai A, Wei L and Zhang F: Immunological network analysis in HPV associated head and neck squamous cancer and implications for disease prognosis. Mol Immunol 96: 28-36, 2018.

14. Lau SK, Chu PG and Weiss LM: CD163: A specific marker of macrophages in paraffin-embedded tissue samples. Am J Clin Pathol 122: 794-801, 2004.

15. Li C, Shintani S, Terakado N, Nakashiro K and Hamakawa H: Infiltration of tumor-associated macrophages in human oral squamous cell carcinoma. Oncol Rep 9: 1219-1223, 2002.

16. Galdiero MR, Bonavita E, Barajon I, Garlanda C, Mantovani A and Jaillon S: Tumor associated macrophages and neutrophils in cancer. Immunobiology 218: 1402-1410, 2013.

17. Chen X, Fu E, Lou H, Mao X, Yan B, Tong F, Sun J and Wei L. IL-6 induced M1 type macrophage polarization increases radiosensitivity in HPV positive head and neck cancer. Cancer Lett 456: 69-79, 2019.

18. Geiger-Maor A, Guedj A, Even-Ram S, Smith Y, Galun E and Rachmilewitz J: Macrophages regulate the systemic response to DNA damage by a cell nonautonomous mechanism. Cancer Res 75: 2663-2673, 2015.

19. Fulcher CD, Haigentz M Jr and Ow TJ; Education Committee of the American Head and Neck Society (AHNS): AHNS Series: Do you know your guidelines? Principles of treatment for locally advanced or unresectable head and necksquamous cell carcinoma. Head Neck 40: 676-686, 2018.

20. Brakenhoff RH, Wagner S and Klussmann JP: Molecular patterns and biology of HPV-associated HNSCC. Recent Results Cancer Res 206: 37-56, 2017.

21. Foy JP, Bazire L, Ortiz-Cuaran S, Deneuve S, Kielbassa J, Thomas E, Viari A, Puisieux A, Goudot P, Bertolus C, et al: A 13-gene expression-based radioresistance score highlights the heterogeneity in the response to radiation therapy across HPV-negative HNSCC molecular subtypes. BMC Med 15: 165, 2017.

22. Pike LRG, Hwang WL, Royce TJ, Sanford NN and Mahal BA: HPV status predicts for improved survival following chemotherapy in metastatic squamous cell carcinoma of the oropharynx. Oral Oncol 86: 69-74, 2018.

23. Ziemann F, Seltzsam S, Dreffke K, Preising S, Arenz A, Subtil FSB, Rieckmann T, Engenhart-Cabillic R, Dikomey E and Wittig A: Roscovitine strongly enhances the effect of olaparib on radiosensitivity for HPV neg. but not for HPV pos. HNSCC cell lines. Oncotarget 8: 105170-105183, 2017.

24. Prevc A, Kranjc S, Cemazar M, Todorovic V, Zegura B, Novak M, Filipic M, Flezar MS, Kirbis IS, Rotter A, et al: Dose-modifying factor of radiation therapy with concurrent cisplatin treatment in HPV-Positive squamous cell carcinoma: A preclinical study. Radiat Res 189: 644-651, 2018.

25. Arenz A, Ziemann F, Mayer C, Wittig A, Dreffke K, Preising S, Wagner S, Klussmann JP, Engenhart-Cabillic R and Wittekindt C: Increased radiosensitivity of HPV-positive head and neck cancer cell lines due to cell cycle dysregulation and induction of apoptosis. Strahlenther Onkol 190: 839-846, 2014.

26. Speel EJ: HPV integration in head and neck squamous cell carcinomas: Cause and consequence. Recent Results Cancer Res 206: 57-72, 2017.

27. Liu YC, Cai ZM and Zhang XJ: Reprogrammed CRISPR-Cas9 targeting the conservedregions of HPV6/11 E7 genes inhibits proliferation and induces apoptosis inE7-transformed keratinocytes. Asian J Androl 18: 475-479, 2016. 
28. Hu Z, Yu L, Zhu D, Ding W, Wang X, Zhang C, Wang L, Jiang X, Shen H, He D, et al: Disruption of HPV16-E7 by CRISPR/Cas system induces apoptosis and growth inhibition in HPV16 positive human cervical cancer cells. Biomed Res Int 2014: 612823, 2014.

29. Moskovitz JM, Moy J, Seiwert TY and Ferris RL: Immunotherapy for head and neck squamous cell carcinoma: A review of current and emerging therapeutic options. Oncologist 22: 680-693, 2017.

30. Wang HC, Chan LP and Cho SF: Targeting the immune microenvironment in the treatment of head and neck squamous cell carcinoma. Front Oncol 9: 1084, 2019.

31. Yahaya MAF, Lila MAM, Ismail S, Zainol M and Afizan NARNM: Tumour-associated macrophages (TAMs) in colon cancer and how to reeducate them. J Immunol Res 2019: 2368249, 2019.

32. Wang J, Gao K, Lei W, Dong L, Xuan Q, Feng M, Wang J, Ye X, Jin T, Zhang Z and Zhang Q: Lymphocyte-to-monocyte ratio is associated with prognosis of diffuse large B-cell lymphoma: Correlation with CD163 positive M2 type tumor-associated macrophages, not PD-1 positive tumor-infiltrating lymphocytes. Oncotarget 8: 5414-5425, 2017.

33. Alves AM, Diel LF and Lamers ML: Macrophages and prognosis of oral squamous cell carcinoma: A systematic review. J Oral Pathol Med 47: 460-467, 2018.

34. Rades D, Seidl D, Janssen S, Strojan P, Karner K, Bajrovic A, Hakim SG, Wollenberg B and Schild SE: Comparing two lower-dose cisplatin programs for radio-chemotherapy of locally advanced head-and-neck cancers. Eur Arch Otorhinolaryngol 274: 1021-1027, 2017.

35. Ozbilgin MK, Aktas C, Uluer ET, Buyukuysal MC, Gareveran MS and Kurtman C: Influence of radiation exposure during radiotherapy. Evidence for the increase of versican and heparin-binding EGF-like growth factor concentrations. Anal Quant Cytopathol Histpathol 38: 126-132, 2016.

36. Gao L, Zhang W, Zhong WQ, Liu ZJ, Li HM, Yu ZL and Zhao YF: Tumor associated macrophages induce epithelial to mesenchyma transition via the EGFR/ERK1/2 pathway in head and neck squamous cell carcinoma. Oncol Rep 40: 2558-2572, 2018.

37. Zhao G, Liu L, Peek RM Jr, Hao X, Polk DB, Li H and Yan F: Activation of epidermal growth factor receptor in macrophages mediates feedback inhibition of M2 polarization and gastrointestinal tumor cell growth. J Biol Chem 291: 20462-20472, 2016
38. Carroll MJ, Kapur A, Felder M, Patankar MS and Kreeger PK: M2 macrophages induce ovarian cancer cell proliferation via a heparin binding epidermal growth factor/matrix metalloproteinase 9 intercellular feedback loop. Oncotarget 7: 86608-86620, 2016.

39. Nabeshima A, Matsumoto Y, Fukushi J, Iura K, Matsunobu T, Endo M, Fujiwara T, Iida K, Fujiwara Y, Hatano M, et al: Tumour-associated macrophages correlate with poor prognosis in myxoid liposarcoma and promote cell motility and invasion via the HB-EGF-EGFR-PI3K/Akt pathways. Br J Cancer 112: $547-555,2015$.

40. Schrevel M, Osse EM, Prins FA, Trimbos JBMZ, Fleuren GJ, Gorter A and Jordanova ES: Autocrine expression of the epidermal growth factor receptor ligand heparin-binding EGF-like growth factor in cervical cancer. Int J Oncol 50: 1947-1954, 2017.

41. Psyrri A, Seiwert TY and Jimeno A: Molecular pathways in head and neck cancer: EGFR, PI3K, and more. Am Soc Clin Oncol Educ Book: 246-255, 2013

42. Normanno N, De Luca A, Bianco C, Strizzi L, Mancino M, Maiello MR, Carotenuto A, De Feo G, Caponigro F and Salomon DS: Epidermal growth factor receptor (EGFR) signaling in cancer. Gene 366: 2-16, 2006.

43. Wang Z: ErbB receptors and cancer. Methods Mol Biol 1652: 3-35, 2017.

44. Kriegs M, Kasten-Pisula U, Rieckmann T, Holst K, Saker J, Dahm-Daphi J and Dikomey E: The epidermal growth factor receptor modulates DNA double-strand break repair by regulating non-homologous end-joining. DNA Repair (Amst) 9: 889-897, 2010.

45. Toulany M, Baumann M and Rodemann HP: Stimulated PI3K-AKT signaling mediated through ligand or radiation-induced EGFR depends indirectly, but not directly, on constitutive K-Ras activity. Mol Cancer Res 5: 863-872, 2007. 